\title{
Sur le mouvement d'une bulle de gaz dans un liquide
}

\section{Gas bubble motion in a liquid medium}

\author{
Raymond Comolet \\ Professeur à l'Université Pierre et Marie Curie \\ Laboratoire de Mécanique Expérimentale des Fluides - Campus d'Orsay 91405
}

\section{Introduction}

Le mouvement libre d'un globule de fluide dans un autre non miscible pose un problème difficile à résoudre car la forme du globule et le comportement de l'interface ne sont pas déterminés à priori. Selon les conditions cette forme change, elle peut être proche de celle d'une sphère, d'un ellipsoïde, d'une calotte sphérique, et l'interface peut se comporter comme une paroi sur laquelle les fluides en présence peuvent glisser plus ou moins.

D'une manière générale on peut dire que la forme géométrique du globule dépend des forces suivantes mises en jeu dans son mouvement : forces d'inertie, de viscosité, de pesanteur, de tension interfaciale, tandis que le comportement de l'interface dépend de l'état de pureté des fluides en présence.

Depuis longtemps on avait observé que le globule revêtait un aspect quasi-sphérique quand il était de petite taille, un aspect ellipsoïdal, ou lenticulaire plus ou moins régulier, quand il augmentait de volume, et, dans le cas des grosses bulles de gaz, l'aspect d'une calotte sphérique (voir l'analyse critique de Benfratello [1], les travaux de Haberman et Morton [2], de Wegener et Parlange [3]). Ces particularités géométriques ont fait l'objet d'un certain nombre de recherches théoriques (analyse de Harper [4]) tandis que les efforts des expérimentateurs se sont portés principalement sur la détermination de la vitesse limite $V$ du globule. C'est ainsi que Wallis [5] a donné un procédé de calcul universel pour déterminer $V$, proposant par exemple, pour les bulles de gaz, une demi-douzaine de formules différentes chacune valable dans un domaine distinct bien défini.

Par ailleurs l'expérience montre que le mouvement du globule est très sensible, surtout quand il est de petite taille, à toute contamination : il s'agit là d'un phénomène difficile à préciser qui a pour conséquences : - d'obliger l'expérimentateur à travailler dans des condi- tions très strictes de pureté chimique des fluides (ce qui n'a pas toujours été le cas)

- de laisser cependant planer quelque incertitude sur la valeur pratique de toute formulation proposée pour déterminer le mouvement des petits globules ${ }^{(1)}$.

Dans ce qui suit nous commencerons par donner un exposé classique sur le mouvement des bulles et des gouttes. Nous continuerons par quelques développements concernant particulièrement le mouvement des bulles et une tentative de représentation universelle de leur vitesse limite. Nous terminerons par quelques réflexions sur deux problèmes non encore résolus posés par la mécanique des bulles.

\section{Paramètres sans dimensions}

Dans un fluide au repos, considérons le mouvement d'un globule de fluide non miscible. Ce mouvement est généralement provoqué par la pesanteur (mais il pourrait l'être par l'action d'un champ de forces quelconque) et le schéma que nous retenons est celui d'une goutte ou d'une bulle ayant atteint sa vitesse limite $V$ (chute libre ou ascension libre). On appellera $d$ le diamètre de la sphère de même volume $v$ que le globule (diamètre de la sphère équivalente). Par définition on a :

$$
v=\pi \frac{d^{3}}{6}
$$

Nous supposerons que les fluides en présence sont incompressibles $^{(2)}$ et newtoniens de sorte que les paramètres physiques qui les caractérisent : masse volumi-

(1) Si une formule est exacte et concerne des interfaces non contaminées, elle ne le sera sans doute plus dans les cas pratiques où les fluides en présence ne sont jamais parfaitement purs.

(2) Par exemple si le milieu ambiant est gazeux, cela revient à supposer que la vitesse $V$ est petite devani la célérité du son. 
que, viscosité, tension interfaciale, ne dépendent que de la température. Nous ferons abstraction des phénomènes de transport de masse ou de chaleur se produisant éventuellement à l'interface et nous appellerons (Fig. 1) :

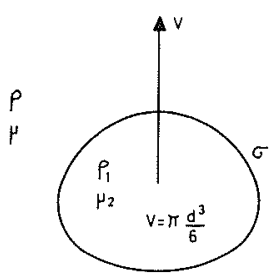

Figure 1

$\mu$ et $\rho$ respectivement la viscosité dynamique et la masse volumique du fluide ambiant

$\mu_{1}$ et $\rho_{1}$ les grandeurs physiques correspondantes du globule

$\sigma \quad$ la tension interfaciale, à la surface du globule $g \quad$ l'accélération de la pesanteur.

La donnée des paramètres indépendants : $d$ (ou $v$ ), $\rho, \mu$, $\rho_{1}, \mu_{1}, \sigma$ et $g$ suffit à déterminer le mouvement libre du globule (dans le champ de pesanteur) et ses caractéristiques : vitesse limite $V$, forme du globule, . .

Par exemple on peut dire que $V$ est une fonction de ces 7 paramètres et écrire :

$$
V=f_{1}\left(d, \rho, \mu, \rho_{1}, \mu_{1}, \sigma, g\right)
$$

ou bien :

$$
g_{1}\left(V, d, \rho, \mu, \rho_{1}, \mu_{1}, \sigma, g\right)=0
$$

Des considérations d'analyse dimensionnelle permettent de présenter cette dernière expression sous une forme adimensionnelle telle que :

$$
\psi\left(\frac{\rho V d}{\mu}, \frac{V^{2}}{d g}, \frac{\rho V^{2} d}{\sigma}, \frac{\rho_{1}}{\rho}, \frac{\mu_{1}}{\mu}\right)=0
$$

soit :

$$
\psi\left(\Re, \mathscr{\vartheta}, \Re 9, \frac{\rho_{1}}{\rho}, \frac{\mu_{1}}{\mu}\right)=0
$$

en introduisant les combinaisons sans dimensions classiques:

$$
\left.\begin{array}{rl}
\mathscr{G} & =\frac{\rho V d}{\mu} \text { nombre de Reynolds } \\
\mathscr{H} & =\frac{V^{2}}{d g} \quad \text { nombre de Froude } \\
\Re ९=\frac{\rho V^{2} d}{\sigma} & \text { nombre de Weber }
\end{array}\right\}
$$

On peut noter que $V$ et $d$ se retrouvent dans les 3 combinaisons $\mathcal{Q}, \widetilde{J^{\prime \prime}}, 2 \%$. De même que dans l'équation 2 où la vitesse $V$ apparaissait comme une fonction de 7 variables indépendantes, de même on peut, dans l'équation 5, expliciter l'une des combinaisons en fonction des 4 autres, par exemple Эु, et écrire :

$$
\mathscr{J}=\phi\left(\mathcal{R}, \Re, \frac{\rho_{1}}{\rho}, \frac{\mu_{1}}{\mu}\right)
$$

\section{Remarques}

1. Avec les 6 paramètres $V, d, \rho, \mu, \sigma, g$ nous avons constitué les trois groupements indépendants sans dimensions $\mathcal{R}, \mathscr{J}, ~ \& 9$. On peut construire bien d'autres groupements sans dimensions, mais ce ne seront jamais que des combinaisons monômes des précédents. Ainsi par exemple :

$\mathscr{O}=\frac{\rho g d^{2}}{\sigma}=\frac{29}{\overparen{\Im}} \quad \begin{aligned} & \text { nombre de Bond, ou d'Eötvös } \\ & \text { ou de Laplace }\end{aligned}$

$\mathscr{L}=\frac{\mu V}{\sigma}=\frac{\Re 9}{\Omega} \quad$ nombre de Levitch

$\mathscr{P}_{0}=\frac{\mu V}{\rho g d^{2}}=\frac{\mathscr{g}}{\mathcal{R}} \quad$ nombre de Poiseuille

$\mathfrak{M}_{0}=\frac{g \mu^{4}}{\rho \sigma^{3}}=\frac{2 \rho^{3}}{\mathscr{H} \Omega^{4}}$ nombre de Morton

On remarquera que $\mathcal{K}_{0}$ ne contient ni $d$, ni $V$, mais seulement les paramètres physiques qui caractérisent les fluides et le champ de forces.

2. Le raisonnement que nous avons fait, supposait essentiellement que le mouvement du globule dépendait de 7 paramètres indépendants ${ }^{(3)}$. Dans certains cas il peut apparaître que l'un ou plusieurs de ces paramètres ne jouent aucun rôle : il $\mathrm{y}$ a lieu alors de les supprimer, ce qui diminue du même nombre celui des combinaisons adimensionnelles indépendantes qu'on peut constituer dans les expressions 4,5 ou 7 .

\section{Coefficient de traînée}

Au lieu de $V$, on pourrait étudier de la même façon, toute caractéristique dimensionnelle ou non, attachée au mouvement du globule. Etudions par exemple le coefficient de traînée $C_{x}$ du globule. Par définition il est donné par la relation :

$$
\left|\rho-\rho_{1}\right| v g=C_{x} \rho s \frac{V^{2}}{2}
$$

qui exprime l'égalité des forces de poussée hydrostatique et des forces de résistance hydrodynamique et visqueuse, $s$ est le maitre-couple du globule, défini dans le sens de $V$. Posons :

$$
\frac{v}{s}=\alpha d
$$

$\alpha$ est un coefficient sans dimension ne dépendant que de la forme géométrique du globule et de son orienta-

(3) La prise en compte d'une éventuelle contamination de l'interface ferait intervenir des paramètres supplémentaires difficiles à préciser. 
tion vis à vis de $V$ (pour un globule sphérique $\alpha=2 / 3$ ). Nous avons:

$$
\frac{V^{2}}{d g}=\frac{\left|\rho-\rho_{1}\right|}{\rho} \frac{2 \alpha}{C_{x}}
$$

soit :

$$
\widetilde{3}=11-\frac{\rho_{1}}{\rho} \mid \frac{2 \alpha}{C_{x}}
$$

Connaissant $\rho_{1} / \rho$ et $\bar{\partial}$, on peut donc calculer $C_{x} / \alpha$. Comme Э̆ lui-même, $C_{x} / \alpha$ est une fonction des 4 paramètres adimensionnels $R, 39, \rho_{1} / p, \mu_{1} / \mu$. On voit en particulier que ce sont les variations de $C_{x} / \alpha$ qui doivent être étudiées, et non celles de $C_{x}$ seul $^{(4)}$.

\section{Vitesse limite des bulles de gaz dans les liquides peu visqueux}

La masse volumique $\rho_{1}$ des gaz et leur viscosité dy. namique $\mu_{1}$ étant généralement bien inférieures aux grandeurs correspondantes des liquides, on sera amenés à faire des simplifications dans les équations. Par exemple en négligeant $\rho_{1} / \rho$ devant l'unité dans les équations 11 et 12 , on écrira :

$$
\text { ซ }=\frac{2 \alpha}{C_{x}}
$$

Deux grands domaines apparaissent quand on fait l'étude de ce mouvement :

- Le domaine I où la búlle est de forme sensiblement sphérique, sa trajectoire étant rectiligne. La tension interfaciale $\sigma$ impose cette forme à la bulle mais n'intervient pas dans le mouvement. On observe que $\rho_{1} / \rho$ et $\mu_{1} / \mu$ n'interviennent pas du tout, et l'équation 13 se réduit à :

$$
\text { 可 }=\Phi_{1}(\text { (R) }
$$

- Le domaine II où l'aspect de la bulle est très variable, passant de la forme d'un ellipsoïde aplati à celle d'une calotte sphérique en passant par des formes lenticulaires plus ou moins régulières. Dans ce domaine, où la trajectoire n'est pas toujours rectiligne, on observe que $\rho_{1} / \rho$ et $\mu_{1} / \mu$ n'interviennent toujours pas et l'expression 13 se réduit à :

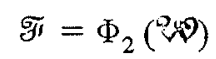

\section{Etude du domaine I : Domaine visqueux}

Quand la bulle atteint sa vitesse limite la valeur de $C_{x} / \alpha$ est déterminée par l'équation 13 , mais $\alpha$ et $C_{x}$ ne le sont séparément que si la forme de la bulle est connue. C'est ce qui se passe dans le cas présent où la bulle est sphérique, $\alpha=2 / 3$, et on peut étudier

(4) Cette restriction résulte évidemment de la définition même de $C_{x}$ : la valeur de $C_{x}$ n'a de signification physique que si la forme du globule est connue. son mouvement à la lumière des connaissances acquises sur celui des sphères. Mais deux hypothèses sont possibles selon que l'interface se comporte comme une paroi rigide ou comme une paroi mobile.

\section{Bulle sphérique à interface rigide}

L'équation 13 s'écrit :

$$
\mathscr{\mathscr { Y }}=\frac{V^{2}}{d g}=\frac{4}{3 C_{x}}
$$

où $C_{x}$ est une fonction de $\mathcal{R}$ donnée par l'expérience ou par la théorie (Fig. 2).

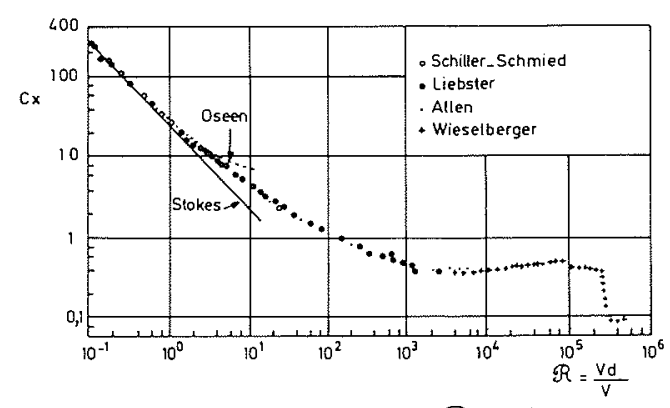

Figure 2 - Variations de $C_{x}$ avec $\mathcal{R}$ (sphère solide)

Quand $\mathcal{R}$ est très petit, $\mathcal{R}<1$ par exemple, on a sensiblement :

$$
C_{x}=\frac{24}{\mathscr{Q}} \text { formule de Stokes }{ }^{(5)}
$$

Dans le domaine $0<R<5000$ on peut utiliser la formule empirique suivante :

$$
C_{x}=\frac{24}{82}\left[1+\frac{82}{R}^{1 / 2}\right]^{2} \text { formule d'Abraham [6] }
$$

Quand $Q$ est assez grand, par exemple $\mathcal{Q}>10^{3}$, mais inférieur à une valeur critique $\mathcal{R}_{c}$ de l'ordre de $10^{5}$ à $2 \times 10^{5}$, on a sensiblement :

$$
C_{x} \cong 0,45
$$

En transportant ces expressions de $C_{x}$ dans l'équation 16 , on obtient des expressions du type (14), qu'il est facile de représenter graphiquement (fig. 3). Les résultats sont particulièrement simples avec la formule de Stokes $(\mathscr{Q}<1)$. On trouve :

$$
\mathscr{\Re}=\frac{\mathfrak{R}}{18}
$$

(5) $\mathrm{La}$ formule de Stokes est une formule théorique établie dans le cas de mouvements rampants, donc pour les très petites valeurs de $Q$. Pour $Q=1$, par exemple, elle donne une valeur de $C_{x}$ trop faible de $14 \%$. Une formule plus précise a été proposée par Oseen :

$$
C_{x}=\frac{24}{\mathscr{Q}}+4,5=\frac{24}{\mathfrak{R}}\left(1+\frac{3}{16} \mathcal{Q}\right) \text { formule de Oseen }
$$

Pour $\mathfrak{R}=1$, cette expression donne une valeur de $C_{x}$ trop forte de $2.5 \%$. 


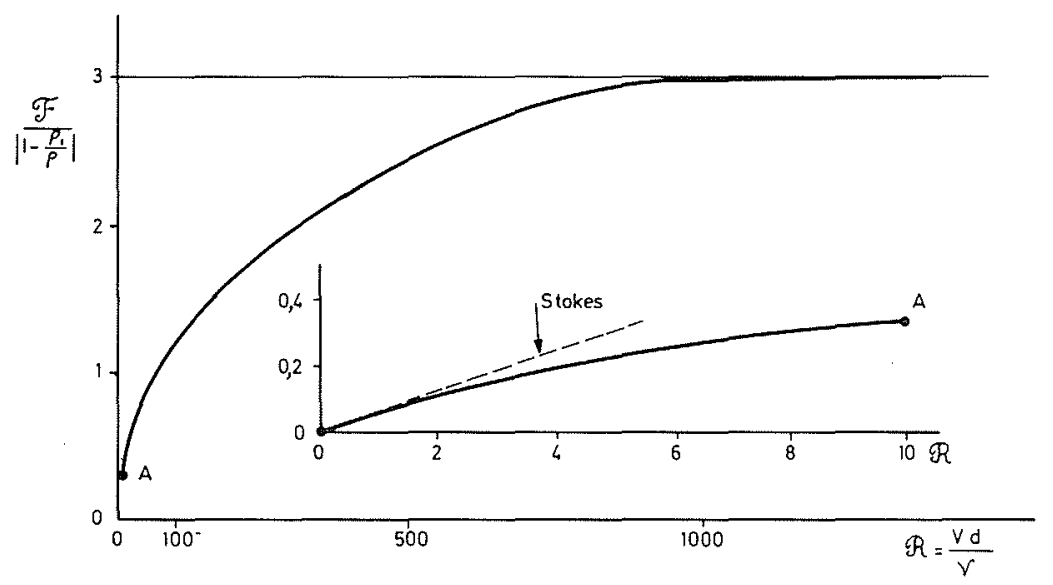

Figure 3 - Sphère solide (Pour une bulle de gaz $\rho_{1} / \rho<<1$ )

soit :

$$
V=\frac{g}{18 v} d^{2}
$$

\section{Bulle sphérique à interface mobile}

Lorsque l'interface de la bulle est mobile, les contraintes tangentielles se conservent de part et d'autre de la sphère ${ }^{(6)}$. Comme il s'agit de gaz, on admet que ces mouvements internes ont un effet négligeable sur celui de la bulle (ce ne serait pas le cas si nous considérions le mouvement d'une goutte de liquide). L'expression 16 est toujours valable et les variations de $C_{x}$ avec $\mathcal{R}$ sont données par les formules théoriques suivantes, dans l'hypothèse où la surface entière de la sphère est mobile. Quand $\mathcal{R}$ est petit, $\mathcal{R}<1$, on a sensiblement :

$$
C_{x}=\frac{16}{\mathcal{R}}^{(7)}
$$

Quand $\mathcal{R}$ est assez grand, mais inférieur à $10^{3}$, on a :

$$
C_{x}=\frac{32}{\Omega}\left(1+\frac{0,814}{\Omega^{1 / 2}}\right)^{(8)}
$$

(6) Dans le cas précédent la rigidité de la paroi faisant écran à ces contraintes et le mouvement de translation de la bulle n'entraînait aucun mouvement intérieur à celle-ci.

(7) Cette formule dérive de l'expression générale donnée par Hadamard [7] et Rybczynski [8] pour une sphère fluide en mouvement rampant :

$$
C_{x}=\frac{24}{\mathcal{R}} \frac{\frac{2}{3}+\frac{\mu_{1}}{\mu}}{1+\frac{\mu_{1}}{\mu}}
$$

(8) Cette formule dérive de l'expression générale donnée par Winnikov et Chao [9] :

$$
C_{x}=\frac{48}{R} \frac{\frac{2}{3}+\frac{\mu_{1}}{\mu}}{1+\left(\frac{\rho_{1}}{\rho} \frac{\mu_{1}}{\mu}\right)^{1 / 2}}\left(1+\frac{0,814}{\Omega^{1 / 2}}\right)
$$

établie en admettant l'existence d'une couche limite extérieure entourant complètement la sphère fluide.
En transportant ces expressions de $C_{x}$ dans l'équation 16 , on obtient encore des expressions du type 14 : $\mathscr{y}=\Phi_{1}(R)$. Les résultats sont particulièrement simples dans le cas où $Q$ est inférieur à 1 , on trouve :

$$
\widetilde{\jmath I}=\frac{\Re}{12}
$$

soit :

$$
V=\frac{g}{12 \nu} d^{2}
$$

\section{Résultats expérimentaux}

Avant d'aller plus loin, il est nécessaire de faire appel à l'expérience pour déterminer les conditions dans lesquelles l'une ou l'autre des deux hypothèses précédentes peut être retenue : sphère à interface rigide ou sphère à interface mobile.

Quand les bulles sont très petites, tous les auteurs s'accordent pour reconnaître à la bulle un comportement de sphère rigide, et ce, jusqu'à un diamètre critique $d_{c}$ qui dépend des conditions expérimentales $d_{c}$ est d'autant plus petit que le liquide ambiant est plus pur. Les expériences très soignées de Gorodetzkaia [10] faites avec des bulles d'air dans de l'eau bidistillée ont donné comme ordre de grandeur : $d_{c}=0,06 \mathrm{~cm}$, $V=7,5 \mathrm{~cm}, \Omega=45$ (Fig. 4). Quand $d$ est inférieur à $0,01 \mathrm{~cm}$, la loi de Stokes s'applique très bien (pour l'eau).

Quand le diamètre de la bulle dépasse la valeur critique $d_{c}$, la vitesse d'ascension croît plus vite que ne l'indique la théorie de la sphère rigide, mais sans confirmer pour autant la théorie de la sphère à paroi mobile : en fait la loi de variation de $V$ avec $d$ se situe entre ces deux cas extrêmes, plus ou moins proche de l'un ou de l'autre selon l'état de pureté du liquide ambiant (Haberman et Morton [2], Gorodetzkaia [10]). S'il s'agit de bulles d'air dans l'eau ordinaire (eau du robinet) la théorie de la sphère rigide s'applique jusqu'à 


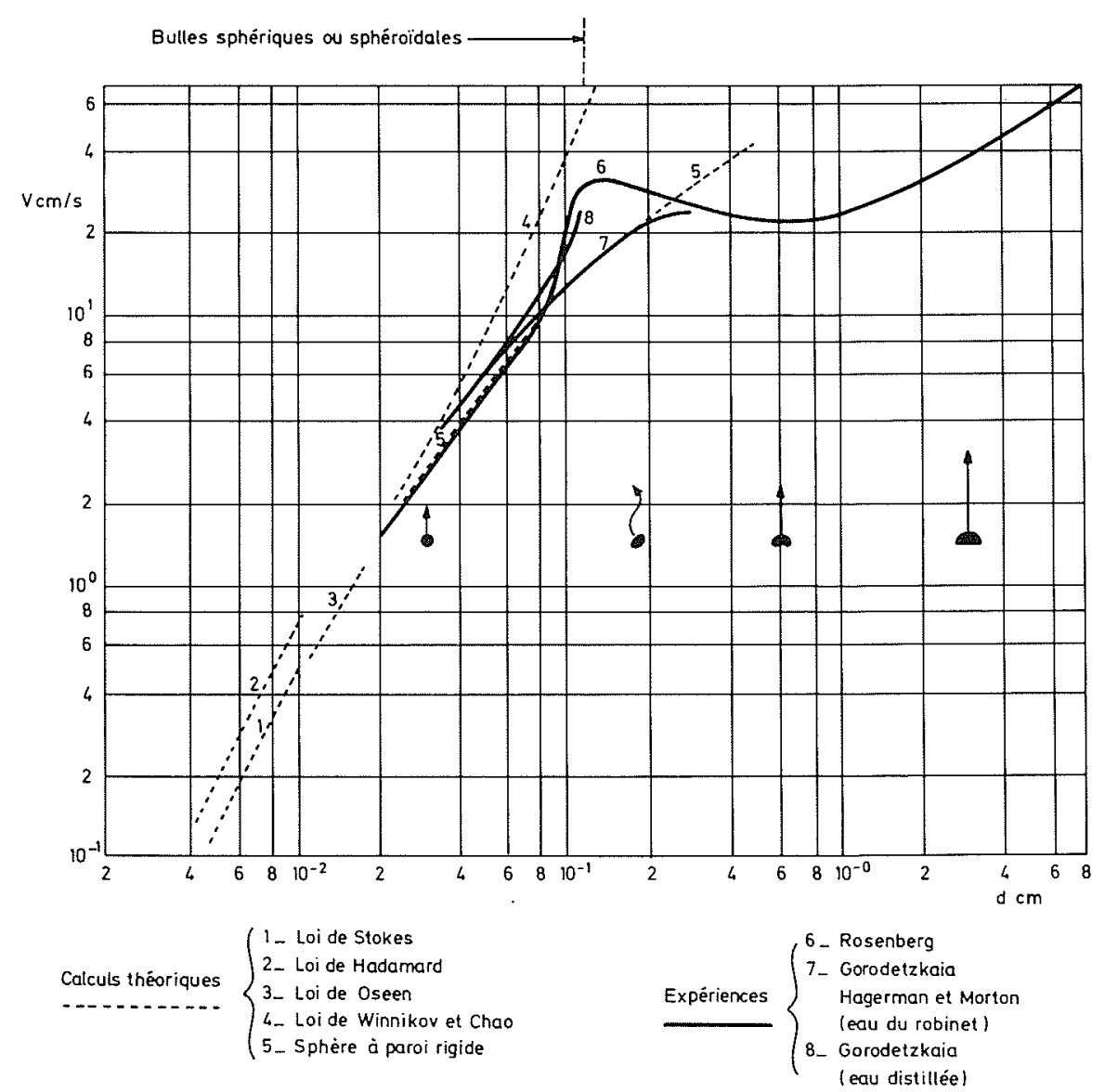

Figure 4 - Vitesse d'ascension limite de bulles d'air dans l'eau

des valeurs de $Q$ de plusieurs centaines (Fig. 4) ${ }^{(9)}$.

\section{Grandeurs caractéristiques}

Le domaine I où la bulle est supposée sphérique, est caractérisée par une expression générale de type 14 : $\mathscr{J I}=\Phi(\mathcal{R})$ qu'on sait expliciter d'une manière plus ou moins approchée. Les deux groupements adimensionnels qui y figurent $\mathscr{\mathscr { Y }}=V^{2} / d g$ et $\Omega=V d / \nu$ sont classiques mais ont l'inconvénient de contenir tous deux $V$ et $d$. D'autres groupements adimensionnels peuvent être utilisés pour étudier le mouvement de la bulle dans ce domaine $I$, en particulier il peut être intéressant de disposer de groupements qui contiennent séparément $V$ et $d$.

En écrivant par exemple :

$$
\left(\frac{\mathcal{R}^{2}}{\mathscr{g}}\right)^{1 / 3}=\left(\frac{d^{3} g}{\nu^{2}}\right)^{1 / 3}=\frac{d}{\left(\frac{\nu^{2}}{g}\right)^{1 / 3}}
$$

on forme une combinaison adimensionnelle nouvelle

(9) On aboutit aux mêmes conclusions quand on étudie le mouvement d'une goutte de liquide dans un autre, non miscible : selon les conditions opératoires la vitesse limite de la goutte obéit à la théorie de la sphère rigide quand $d$ est assez petit ou si les liquides présentent une certaine contamination ; mais cette vitesse limite se rapproche de celle que donne la théorie de la sphère à paroi mobile, quand les liquides sont purs. ne contenant que $d$ :

$$
\frac{d}{\left(\frac{\nu^{2}}{g}\right)^{\frac{1}{3}}}=\frac{d}{d}
$$

qui permet de comparer le diamètre $d$ de la bulle à une dimension linéaire $\left(v^{2} / g\right)^{1 / 3}$ constituée avec les propriétés physiques du milieu ambiant environnant la bulle. En posant :

$$
d_{1}=\left(\frac{\nu^{2}}{g}\right)^{1 / 2}
$$

$d_{1}$ apparaît comme une longueur de référence caractéristique de ce milieu ambiant.

De même en écrivant :

$$
(\mathscr{R})^{1 / 3}=\left(\frac{V^{3}}{\nu g}\right)^{1 / 3}=\frac{V}{(\nu g)^{1 / 3}}
$$

on forme une combinaison adimensionnelle nouvelle ne contentant que $V$ :

$$
\frac{V}{(\nu g)^{1 / 3}}=\frac{V}{V_{1}}
$$

dans laquelle la vitesse $V$ de la bulle est comparée à une grandeur cinématique $(\nu g)^{1 / 3}$ homogène à une 
vitesse, constituée avec les propriétés physiques du milieu ambiant. En posant :

$$
V_{1}=(\nu g)^{1 / 3}
$$

$V_{1}$ apparaît comme une vitesse de référence caractéristique de ce milieu ambiant ${ }^{(10)}$.

On a d'ailleurs :

$$
\begin{gathered}
\mathscr{J}=\frac{\left(\frac{V}{V_{1}}\right)^{2}}{\frac{d}{d_{1}}} \\
\mathcal{R}=\frac{V}{V_{1}} \times \frac{d}{d_{1}}
\end{gathered}
$$

On peut ainsi, pour étudier le mouvement de la bulle, remplacer l'expression adimensionnelle $\mathscr{J} I=\Phi_{1}(\mathcal{R})$ par une autre expression adimensionnelle de la forme :

$$
V_{1}=\Phi_{1}\left(\frac{d}{d_{1}}\right)
$$

dont la signification physique est plus simple. On peut vérifier que :

$$
\begin{gathered}
\text { ף }=\frac{V_{1}^{2}}{d_{1} g}=1 \\
\mathcal{R}_{1}=\frac{V_{1} d_{1}}{\nu}=1 \text { soit } V_{1} d_{1}=\nu
\end{gathered}
$$

Ces dernières relations indiquent que, pour une bulle de diamètre $d_{1}$ se déplaçant à la vitesse $V_{1}$, les forces d'inertie, de viscosité et de gravité mises en œuvre sont du même ordre de grandeur.

\section{Exemple}

Quand $\mathcal{Q}$ est assez petit, on a :

$$
\frac{V}{V_{1}}=\frac{1}{18}\left(\frac{d}{d_{1}}\right)^{2}
$$

d'où l'on tire en utilisant $(40)$ :

$$
\frac{V d}{V_{1} d_{1}}=\frac{V d}{\nu}=\frac{1}{18}\left(\frac{d}{d_{1}}\right)^{3}
$$

La condition $\mathcal{R} \leqslant 1$ se traduit donc par la relation :

$$
\frac{d}{d_{1}} \leqslant 18^{1 / 3}=2,6207
$$

(10) On pourrait aussi constituer un temps de référence caractéristique :

$$
t_{1}=\left(\frac{v}{g^{2}}\right)^{1 / 3}
$$

Pour des bulles de gaz dans l'eau, on a :

$$
\begin{aligned}
& d_{1}=4,64 \cdot 10^{-3} \mathrm{~cm} \\
& V_{1}=2,15 \mathrm{~cm} / \mathrm{s}
\end{aligned}
$$

\section{Etude du domaine II : Domaine capillaire}

Le domaine II est celui pour lequel la bulle n'est plus assimilable à une sphère (fig. 4), sa forme passant progressivement, quand $d$ augmente, d'un ellipsoïde aplati à une calotte sphérique de proportions assez bien définies.

\section{Résultats expérimentaux}

Dans ce domaine, les variations de $V$ avec $d$ peuvent être représentées par une loi du type $\mathscr{Y}=\Phi_{2}(29)$ où n'intervient pas le nombre de Reynolds (ni $\rho_{1} / \rho$ et $\left.\mu_{1} / \mu\right)^{(11)}$. La vitesse d'ascension ne dépend plus de la viscosité du fluide ambiant. Elle dépend surtout de la tension interfaciale $\sigma$ (Malenkov [11], Mendelson [12], Comolet [13]). Dans ce domaine, et sauf quand la bulle est assimilable à une calotte sphérique, l'étude des variations de $C_{x}$ ne semble pas d'un grand intérêt, en raison de l'indétermination de sa forme géométrique, souvent variable et instable.

Considérons par exemple la formule proposée par l'auteur [13] :

$$
V=\left(0,52 g d+2,14 \frac{\sigma}{\rho d}\right)^{1 / 2}
$$

Sous forme adimensionnelle, elle s'écrit :

$$
\frac{0,52}{\mathscr{J}}+\frac{2,14}{2 \Omega^{n}}=1
$$

qui est bien du type $\mathscr{Y}_{1}=\phi,(\& 9)$, ou bien encore, en introduisant le nombre de Bond $U 3=\rho g d^{2} / \sigma$ :

$$
2 \curvearrowleft=0,52 \mathscr{B}+2,14
$$

L'une ou l'autre de ces expressions représente bien les résultats expérimentaux effectués avec des liquides peu visqueux (Fig. 5).

\section{Grandeurs caractéristiques}

Dans ce domaine II où s'applique une loi de la forme $\mathscr{I}=\Phi_{2}(\$ 9)$, on peut rechercher, comme cela a été fait dans l'étude du domaine I, une représentation utilisant deux combinaisons sans dimensions qui contiennent séparément $V$ et $d$. Il vient ainsi :

$$
\left(\frac{\Re 9}{\bar{g}}\right)^{1 / 2}=\left(\frac{d^{2}}{\frac{\sigma}{\rho g}}\right)^{1 / 2}=\frac{d}{d_{2}}
$$

(11) Il n'y a pas de théorie générale justifiant une telle loi. Différentes hypothèses de calcul permettent cependant de la proposer. 


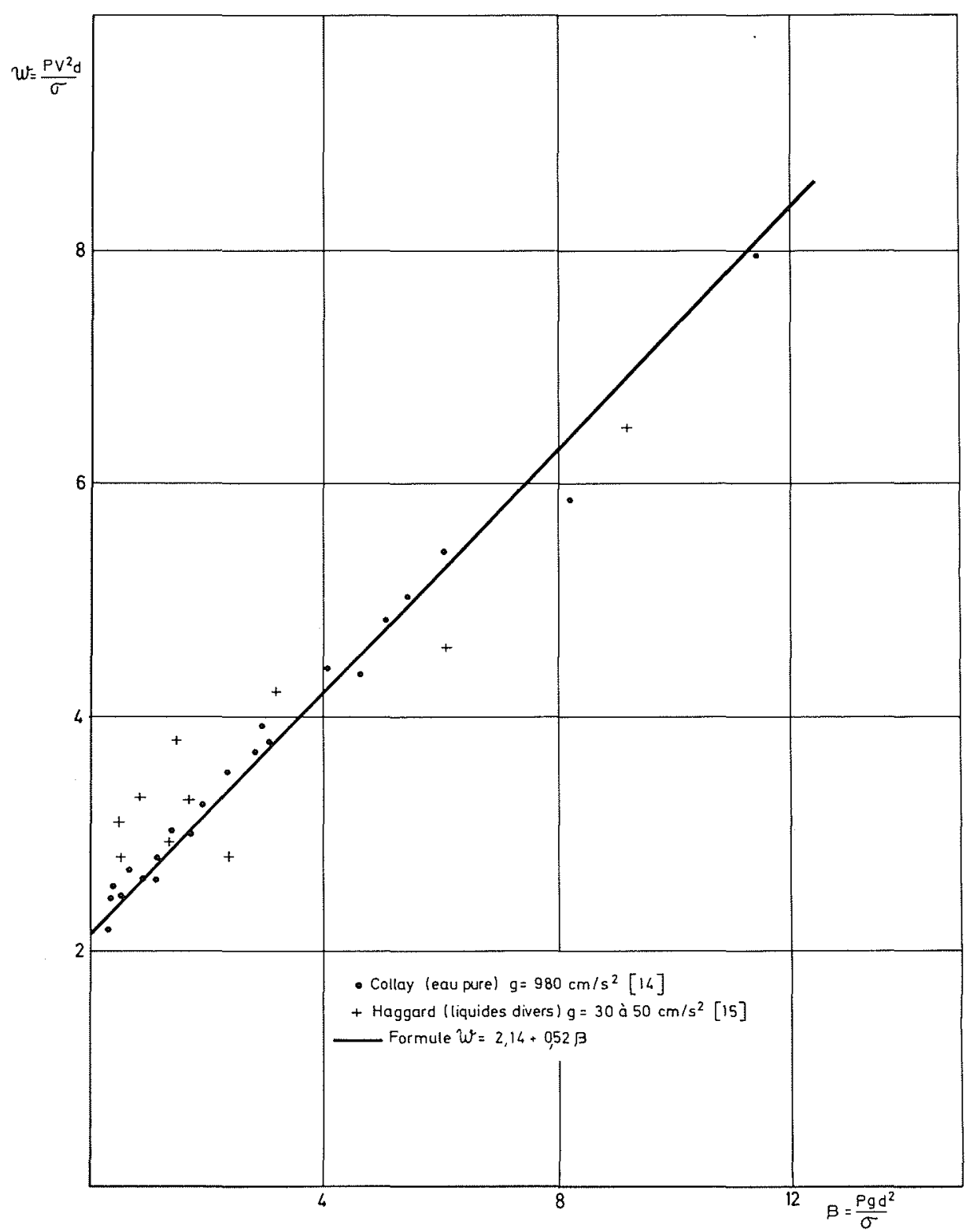

Figure 5 - Etude du domaine II

$$
(\mathscr{H} \leftrightarrow 9)^{1 / 4}=\left(\frac{V^{4}}{\frac{\sigma g}{\rho}}\right)^{1 / 4}=\frac{V}{V_{2}}
$$

en posant :

$$
\begin{aligned}
& d_{2}=\left(\frac{\sigma}{\rho g}\right)^{1 / 2} \\
& V_{2}=\left(\frac{\sigma g}{\rho}\right)^{1 / 4}
\end{aligned}
$$

Nous formons ainsi, avec les 3 paramètres physiques $\rho, \sigma, g$ qui caractérisent le milieu ambiant, une longueur de référence caractéristique $d_{2}$ et une vitesse de référence caractéristique $V_{2}$ auxquelles nous rap- portons respectivement $d$ et $V^{(12)}$.

Dans le domaine II, le mouvement de la bulle peut donc aussi être étudié au moyen d'une expression adimensionnelle de la forme :

$$
\frac{V}{V_{2}}=\phi_{2}\left(\frac{d}{d_{2}}\right)
$$

(12) On pourrait aussi constituer un temps de référence caractéristique :

$$
t_{2}=\left(\frac{\sigma}{\rho g^{3}}\right)^{1 / 4}
$$

(13) En fait, la relation 43 est une expression dimensionnelle de ce type. 
On peut alors vérifier que :

$$
\begin{gathered}
\Im=\frac{V^{2}}{d g}=\frac{\left(\frac{V}{\dot{V}}\right)^{2}}{\frac{d}{d_{2}}} \\
\Re 9=\frac{\rho V^{2} d}{\sigma}=\left(\frac{V}{V_{2}}\right)^{2} \frac{d}{d_{2}}
\end{gathered}
$$

et que :

$$
\begin{gathered}
\mathscr{J}_{2}=\frac{V_{2}^{2}}{d_{2} g}=1 \\
\Re_{2}=\frac{\rho V_{2}^{2} d_{2}}{\sigma}=1
\end{gathered}
$$

Ces deux dernières relations indiquent que, pour une bulle de diamètre $d_{2}$ se déplaçant avec la vitesse $V_{2}$, les forces d'inertie, de viscosité et de capillarité mises en oeuvre sont du même ordre de grandeur.

Dans le domaine II on peut ainsi remplacer l'expression 43 par la suivante :

$$
\frac{V}{V_{2}}=\left(0,52 \frac{d}{d_{2}}+\frac{2,14}{d / d_{2}}\right)^{1 / 2}
$$

\section{Exemple}

Pour des bulles de gaz dans l'eau, on a :

$$
\begin{aligned}
d_{2} & =0,273 \mathrm{~cm} \\
V_{2} & =16,35 \mathrm{~cm} / \mathrm{s}
\end{aligned}
$$

\section{Relations entre les grandeurs caractéristiques. Représentation adimensionnelle universelle}

En résumé :

Dans le domaine I, les paramètres physiques qui interviennent dans le mouvement de la bulle sont $\rho, \mu$ $(\mathrm{ou} \nu), g$ et les grandeurs caractéristiques sont :

$$
\begin{gathered}
d_{1}=\left(\frac{\nu^{2}}{g}\right)^{1 / 3}=\left(\frac{\mu^{2}}{\rho^{2} g}\right)^{1 / 3} \\
V_{1}=(\nu g)^{1 / 3}=\left(\frac{\mu}{\rho} g\right)^{1 / 3}
\end{gathered}
$$

Dans ce domaine le mouvement de la bulle peut être étudié au moyen d'une loi universelle telle que :

$$
\frac{V}{V_{1}}=\phi_{1}\left(\frac{d}{d_{1}}\right)
$$

Quand $d<d_{c}$, la bulle se comporte comme une sphère rigide. Si $Q \leqslant 1$, la loi prend la forme explicite (41) :

$$
\frac{V}{V_{1}}=\frac{1}{18}\left(\frac{d}{d_{1}}\right)^{2}
$$

$\mathrm{Si} R>1$, la loi prend une forme implicite assez bien définie. Quand $d>d_{c}$, l'expression de la loi n'est pas connue, et il est possible qu'une formulation telle que (59) soit insuffisante.

Dans le domaine II, les paramètres physiques qui interviennent dans le mouvement de la bulle sont $\rho$, $\sigma, g$, et les grandeurs caractéristiques sont :

$$
\begin{aligned}
& d_{2}=\left(\frac{\sigma}{\rho g}\right)^{1 / 2} \\
& V_{2}=\left(\frac{\sigma g}{\rho}\right)^{1 / 4}
\end{aligned}
$$

et on a la loi universelle :

$$
\frac{V}{V_{2}}=\phi_{2}\left(\frac{d}{d_{2}}\right)^{(14)}=\left[0,52 \frac{d}{d_{2}}+2,14\left(\frac{d}{d_{2}}\right)^{-1}\right]^{1 / 2}
$$

Ces deux lois universelles 59 et 63 utilisent des grandeurs caractéristiques différentes $d_{1}$ et $d_{2}, V_{1}$ et $V_{2}$, il est naturel de comparer ces dernières. Ainsi :

$$
\begin{aligned}
& \frac{d_{1}}{d_{2}}=\left(\frac{g \mu^{4}}{\rho \sigma^{3}}\right)^{1 / 6}=\pi_{0}^{1 / 6} \\
& \frac{V_{1}}{V_{2}}=\left(\frac{g \mu^{4}}{\rho \sigma^{3}}\right)^{1 / 12}=\pi_{0}^{1 / 12}
\end{aligned}
$$

où $\Re \tau_{0}=g \mu^{4} / \rho \sigma^{3}$ est le nombre de Morton défini en (8) : c'est un nombre sans dimensions caractéristiques du fluide ambiant. Ainsi pour l'eau, nous avons:

$$
\begin{aligned}
& \pi_{0}=2,52 \cdot 10^{-11} \\
& \frac{d_{1}}{d_{2}}=0,131 \\
& \frac{V_{1}}{V_{2}}=0,017
\end{aligned}
$$

Nous pouvons écrire :

$$
\begin{aligned}
& \frac{d}{d_{1}}=\frac{d}{d_{2}} \times \frac{d_{2}}{d_{1}}=\frac{d}{d_{2}} \boldsymbol{K}_{0}^{-1 / 6} \\
& \frac{V}{V_{1}}=\frac{V}{V_{2}} \times \frac{V_{2}}{V_{1}}=\frac{V}{V_{2}} \boldsymbol{K}_{0}^{-1 / 12}
\end{aligned}
$$

On peut donc remplacer la loi 59 du domaine I par :

$$
\frac{V}{V_{2}}=\pi_{0}^{1 / 12} \phi_{1}\left(\frac{d}{d_{2}} \pi_{0}^{-1 / 6}\right)
$$

et pour $Q \leqslant 1$, la loi 60 par :

$$
\frac{V}{V_{2}}=\frac{\pi-1 / 4}{18}\left(\frac{d}{d_{2}}\right)^{2}
$$

(14) On remarquera que $d / d_{2}=(\mathscr{B})^{1 / 2}$ 
Calculons maintenant le nombre de Reynolds. En utilisant (40) il vient :

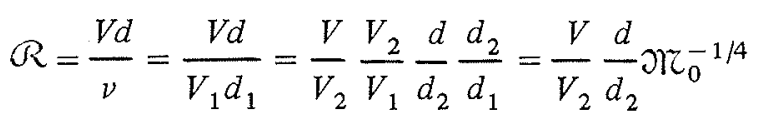

En éliminant $\mathfrak{T C}_{0}$ entre (69) et $(70)$, nous obtenons :

$$
\left(\frac{V}{V_{2}}\right)^{2}=\frac{\mathcal{R}}{18} \frac{d}{d_{2}}
$$

La loi 69 n'étant valable que pour $\mathcal{R}<1$, cette condition se traduit par :

$$
\left(\frac{V}{V_{2}}\right)^{2} \leqslant \frac{1}{18} \frac{d}{d_{2}}
$$

Grâce à ce changement de variables, nous pouvons maintenant représenter l'ensemble des lois définissant la vitesse de la bulle dans le même système de coordonnées.

En utilisant des coordonnées logarithmiques et en posant :

$$
X=\log \frac{d}{d_{2}}
$$

(15) Un autre calcul, traduisant immédiatement la condition 42, donnerait :

$$
\frac{d}{d_{2}} \leqslant 2,620 \operatorname{Rr}_{9}^{1 / 6}
$$

$$
Y=\log \frac{V}{V_{2}}
$$

la loi 68 qui caractérise le domaine I, est représentée par une famille de courbes qu'on peut tracer point par point pour chaque valeur de $\mathfrak{T C}_{0}$

Tant qu'on est au-dessous de la droite de pente $\frac{1}{2}$ (définie par la condition 72) :

$$
Y=\frac{1}{2} \log \frac{1}{18}+\frac{X}{2}=\log 0,236+\frac{X}{2}
$$

ces courbes se confondent avec les droites de pente 2 (définies par la loi 69):

$$
Y=\log \frac{\pi_{0}^{-1 / 4}}{18}+2 X
$$

droites qui se déduisent les unes des autres par une translation parallèle à $Y$, fonction de $\mathfrak{K C}_{0}$ seulement.

Dans ce même système de coordonnées logarithmiques la loi 63 qui caractérise le domaine II est représentée par une courbe unique, présentant un minimum correspondant à :

$$
\begin{aligned}
& \frac{d}{d_{2}}=2,028 \\
& \frac{V}{V_{2}}=1,452
\end{aligned}
$$

Cette courbe est asymptotique d'un côté à la droite

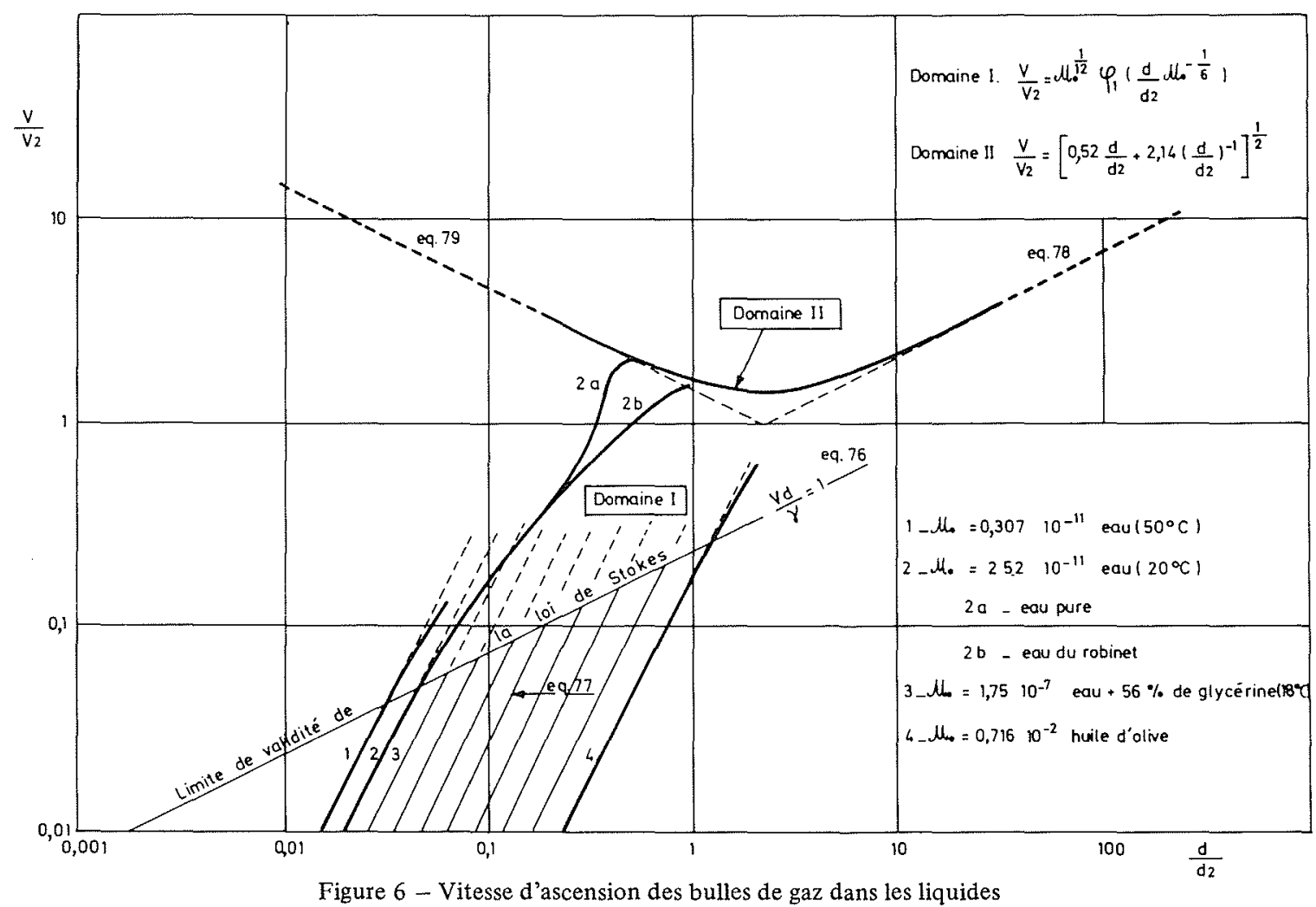


de pente $\frac{1}{2}$ :

$$
Y=\frac{1}{2} \log 0,52+\frac{X}{2}=\log 0,721+\frac{X}{2}
$$

et de l'autre côté à la droite de pente $-\frac{1}{2}$ :

$$
Y=\frac{1}{2} \log 2,14-\frac{X}{2}=\log 1,463-\frac{X}{2}
$$

L'ensemble de ces résultats a été reporté sur la figure 6 qui fournit une représentation universelle pour l'étude de la vitesse limite $V$ des bulles de gaz dans n'importe quel liquide. Dans le domaine $I$, on a une famille de courbes paramétrées par $\boldsymbol{N r}_{0}$; dans le domaine II, on a une courbe unique.

\section{Quelques réflexions sur deux problèmes non résolus}

Deux questions se sont posées au cours de cette étude et qui n'ont pas encore reçu de réponse :

- l'une concerne le passage de l'interface rigide à l'interface mobile

- l'autre concerne le passage de la forme sphérique à la forme elliptique.

Elles appellent cependant les quelques réflexions qui suivent :

\section{Calcul de l'interface}

Quand la bulle est petite et sphérique, son mouvement est analogue à celui d'une sphère solide, et on admet que l'interface se comporte comme une paroi rigide, le liquide adhérent à cette paroi. Une telle analogie ne peut être retenue quand le diamètre $d$ de la bulle dépasse une valeur critique $d_{c}$ qui dépend des conditions expérimentales. On admet alors qu'une partie au moins de l'interface a un comportement différent, le liquide pouvant glisser contre elle (interface mobile). On admet que ce changement de comportement est lié à la présence d'impuretés qui ont des propriétés tensioactives, et qui sont absorbées à l'interface formant une sorte de bouclier comme le ferait une membrane souple. D'après Frumkin et Levich, ces agents tensioactifs sont adsorbés sur le front amont du globule et sont entraînés sur le front arrière par le mouvement du globule créant ainsi un gradient de produit tensioactif grad $\vec{\sigma}$ à la surface du globule. Ce gradient provoquerait des forces tangentielles qui s'opposeraient au mouvement du globule.

Considérons un petit élément $d S$ de l'interface, de centre $M$, et les forces agissant dans le plan tangent en $M$. Si cet élément est en repos relatif par rapport au globule, on a :

$$
\vec{\tau}_{i}=\vec{\tau}_{e}+\operatorname{grad} \vec{x}
$$

les tensions $\vec{\tau}_{i}$ et $\vec{\tau}_{e}$ sont les tensions visqueuses développées respectivement par le fluide intérieur et par le fluide extérieur. La tension $\vec{\tau}$ a un module de la forme $k_{1} \mu V / d$ où $k_{1}$ est un coefficient sans dimensions de l'ordre de l'unité, qui varie avec la position du point $M$ à la surface du globule. Comme il s'agit ici d'une bulle de gaz on peut négliger $\overrightarrow{\tau_{i}}$. Le terme grad $\vec{\sigma}$ caractérise l'influence des variations de $\sigma$ autour de la bulle, son module est de la forme $k_{2} \Delta \sigma / d$ où $k_{2}$ est un coefficient sans dimensions de l'ordre de l'unité et qui varie avec $M, \Delta \sigma$ étant l'amplitude extrême de la variation de $\sigma$ autour de sa valeur moyenne $\sigma_{m}$ (on admettra que $\left.\Delta \sigma \ll \sigma_{m}\right)$. L'équation 80 conduit donc à une expression de la forme :

$$
0=k_{1} \frac{\mu V}{d}+k_{2} \frac{\Delta \sigma}{d}
$$

soit :

$$
\frac{\mu V}{\Delta \sigma}=-\frac{k_{2}}{k_{1}}
$$

L'immobilité relative de $d S$ par rapport à la bulle est assurée, interface rigide, quand cette relation est vérifiée, et $\Delta \sigma$ s'ajuste en fonction de $V$ pour qu'il en soit ainsi.

Nous avons supposé que $\Delta \sigma$ restait petit devant $\sigma_{m}$, de sorte que si $V$ est trop grand, l'équation 81 ne pourra plus être satisfaite. Si donc on appelle $\Delta \sigma_{m}$ la valeur maximale que peut prendre $\Delta \sigma,\left(\Delta \sigma_{m}\right.$ dépend des impuretés et produits tensioactifs présents dans le liquide), il existe une valeur maximale de $V$ (donc de $d$ ), au-delà de laquelle la rigidité de l'interface disparaît. Il existerait ainsi une valeur critique $\mu \mathrm{V} / \Delta \sigma_{m}$ au-delà de laquelle la rigidité de l'interface disparaitrait ${ }^{(16)}$. Si $\Delta \sigma_{m}$ n'est pas très petit, la mobilité de l'interface n'apparaitra que pour des vitesses $V$ notables, c'est-à-dire pour des bulles de dimension notable. $\mathrm{Au}$ contraire, si $\Delta \sigma_{m}$ est très petit, le phénomène apparaîtra pour les très petites bulles.

Pour un liquide parfaitement pur, $\Delta \sigma=0$, la relation 81 ne peut jamais être satisfaite et l'interface n'est rigide en aucun de ses points. La théorie d'Hadamard-Rybizynski pour les petits nombres de Reynolds, celle de Winnikov et Chao pour les grands, devraient donc s'appliquer. Nous avons dit que l'expérience n'a pas réussi à confirmer ces théories, sans doute parce qu'il est très difficile d'obtenir des liquides ayant le degré de pureté requis ; les formules qu'elles proposent peuvent donc être considérées comme donnant des limites supérieures à la vitesse d'ascension des bulles.

\section{Passage de la forme sphérique à la forme elliptique}

En projection sur la normale en $M$, les forces qui agissent sur l'élément $d s$ conduisent à l'équation :

$$
p_{i}=p_{e}+p_{c}
$$

$p_{i}$ est la pression intérieure de la bulle, $p_{e}$ la pression

(16) La disparition de la rigidité n'affecte probablement pas brutalement toute l'interface, elle doit sans doute se manifester d'une manière progressive dans les zones où la condition $\overrightarrow{\tau_{e}}+\operatorname{grad} \vec{\sigma}=0$ ne peut plus être satisfaite. 
extérieure régnant en $M, p_{c}$ étant la pression capillaire, celle qui résulte de l'action de la tension superficielle $\sigma$. Nous avons :

$$
p_{c}=\sigma\left(\frac{1}{R_{1}}+\frac{1}{R_{2}}\right)
$$

où $R_{1}$ et $R_{2}$ sont les rayons de courbure principaux en $M$. Pour un liquide parfaitement pur, $\sigma$ ne dépend pas de $M$ et est constant. (S'il n'en est pas ainsi on supposera, comme nous l'avons fait précédemment, que $\sigma$ varie peu autour de sa valeur moyenne $\sigma_{m}$ ).

Par ailleurs, on peut supposer que $p_{e}$ est la somme de trois termes:

$$
p_{e}=p_{h}+p_{d}+p_{v}
$$

$p_{h}$ est une pression hydrostatique de la forme :

$$
p_{h}=p_{0}+\beta \rho d
$$

$p_{d}$ est une pression dynamique de la forme :

$$
p_{d}=\gamma \rho V^{2}
$$

$p_{v}$ est une pression visqueuse de la forme :

$$
p_{v}=\delta \mu \frac{V}{d}
$$

Les coefficients $\beta, \gamma, \delta$ sont sans dimensions, ils sont de l'ordre de l'unité et varient avec la position de $M$ autour de la bulle. $p_{0}$ est la pression hydrostatique définie dans un plan de référence, par exemple celui qui passe par le centre de la bulle. Pour une position donnée de la bulle, $p_{0}$ est une constante qui ne dépend pas de $M$. On écrira donc (82) sous la forme :

$$
\begin{aligned}
& p_{i}-p_{0}=\sigma_{m}\left(\frac{1}{R_{1}}+\frac{1}{R_{2}}\right)+\beta \rho g d+\gamma \rho V^{2}+\delta \mu \frac{V}{d} \\
& =\frac{\sigma_{m}}{d}\left[\left(\frac{d}{R_{1}}+\frac{d}{R_{2}}\right)+\beta \frac{\rho g d^{2}}{\sigma_{m}}+\gamma \frac{\rho V^{2} d}{\sigma_{m}}+\delta \mu \frac{V}{\sigma_{m}}\right]
\end{aligned}
$$

soit :

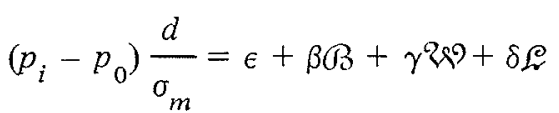

en posant :

$$
\epsilon=\frac{d}{R_{1}}+\frac{d}{R_{2}}
$$

Dans l'expression 89, et pour une position donnée de la bulle, le premier membre est constant ainsi que les nombres sans dimensions $\mathscr{B}, 2 \mathscr{O}$ et $\mathscr{P}$.

Pour que la bulle soit parfaitement sphérique, il faut qu'en tout point $M$ on ait $R_{1}=R_{2}=d / 2$. Dans ces conditions $\epsilon=4$ et l'équation 89 s'écrit :

$$
\beta\left(B+\gamma 2 \Re+\delta \mathscr{P}=\left(p_{i}-p_{0}\right) \frac{d}{\sigma_{m}}-4\right.
$$

Or il n'y a aucune raison de supposer que la somme $\beta \mathscr{B}+\gamma 39+\delta \mathscr{P}$ soit constante et indépendante de la position de $M$, ce qui montre qu'une sphéricité rigoureuse ne se produit probablement pas.

Toutefois, si pour une bulle de diamètre $d$, on a :

$$
\beta \mathfrak{B}+\gamma^{2 \mathfrak{P}}+\delta \mathfrak{P} \ll 1
$$

l'équation 89 conduit à écrire :

$$
\frac{1}{R_{1}}+\frac{1}{R_{2}} \# \frac{p_{i}-p_{e}}{\sigma_{m}}=\mathrm{C}^{\mathrm{te}}
$$

Pour $R_{1} \# R_{2} \# \frac{d}{2}$ cette relation est satisfaite, ce qui montre qu'une quasi-sphéricité de la bulle est possible. $\mathrm{Si}$ la condition 92 n'est pas vérifiée, la variation de

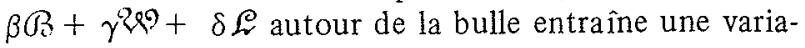
tion correspondante de $\epsilon$, c'est-à-dire une variation non négligeable de la courbure moyenne de la surface de la bulle : celle-ci n'est plus sphérique. Dans ce cas l'un au moins des trois facteurs adimensionnels $\mathcal{B}, 2 \%$, $f$ est assez grand et atteint, par exemple, une valeur critique qui reste à déterminer.

Or ces trois facteurs ne sont pas indépendants, ils sont liés par l'équation du mouvement de la bulle qui établit un rapport entre $V, d, \rho, g$ et $\sigma_{m}$. Ecrivons (89) sous la forme :

$$
\begin{aligned}
\left(p_{i}-p_{0}\right) \frac{d}{\sigma_{m}} & =\epsilon+\mathfrak{B}\left[\beta+\gamma \frac{\mathfrak{Q}}{\mathfrak{B}}+\delta \frac{\mathfrak{S}}{\mathfrak{B}}\right] \\
& =\epsilon+\mathfrak{B}\left[\beta+\gamma \widetilde{\mathscr{H}}+\delta \frac{\mathscr{\mathscr { H }}}{\mathfrak{R}}\right]
\end{aligned}
$$

Tant que la bulle peut être assimilable à une sphère rigide, on sait que $\mathscr{G 1}$ ne dépend que de $\mathcal{R}$ (Fig. 3) : $\mathscr{J}$ croît régulièrement de 0 à 3 quand $\mathcal{R}$ augmente, et Э̆/ $/ R$ diminue régulièrement à partir de $1 / 18$. Dans l'équation 94 , le crochet ne dépend donc que de $R$ et sa valeur numérique est toujours de l'ordre de l'unité, par conséquent la somme $(\mathcal{B}[\beta+\gamma \mathscr{\mathscr { U }}+\delta \mathscr{Y H} / \mathcal{Q}]$ est toujours de l'ordre de grandeur de $\mathscr{O}$. Si $\mathscr{O}$ est petit, la bulle reste donc sensiblement sphérique (la condition 92 est satisfaite). La perte de sphéricité apparaîtra quand $\mathscr{B}$ sera assez grand et atteindra par exemple une certaine valeur critique $\mathfrak{B}_{c^{\prime}}$ (d'après les expérimentateurs cela correspondrait à la frontière entre les domaines I et II). Dans le cas de bulles d'air dans l'eau pure, la perte de sphéricité apparaît aux environ de $d=1,2 \mathrm{~mm}[2-13]$, ce qui donne $\mathbb{B}=0,193$. On peut donc proposer comme critère de perte de sphéricité :

$$
\mathscr{B}=0,2
$$

ce qui correspond à peu près à $d / d_{2}=0,44$

\section{Conclusions}

Au moyen des paramètres physiques $\rho, \mu, \sigma, g$ qui caractérisent le milieu extérieur, on peut constituer diverses grandeurs caractéristiques homogènes res- 
pectivement à une longueur et à une vitesse :

ainsi $d_{1}$ et $V_{1}$ à partir de $\rho, \mu, g$ ainsi $d_{2}$ et $V_{2}$ à partir de $\rho, \sigma, g$

Mais on peut aussi partir des paramètres $\mu, \rho, g$ et constituer :

$$
\begin{aligned}
& d_{3} \doteq \frac{\mu^{2}}{\rho \sigma} \text { homogène à une longueur } \\
& V_{3}=\frac{\sigma}{\mu} \quad \text { homogène à une vitesse }
\end{aligned}
$$

Les trois longueurs caractéristiques $d_{1}, d_{2}, d_{3}$ sont proportionnelles, le coefficient de proportionnalité étant une puissance du nombre de Morton $\Re_{0}$. Il en est de même pour les trois vitesses caractéristiques $V_{1}$, $V_{2}, V_{3}$. Ainsi :

$$
\begin{array}{ll}
\frac{d_{1}}{d_{2}}=\operatorname{NC}_{0}^{1 / 6} & \frac{V_{1}}{V_{2}}=\operatorname{MK}_{0}^{1 / 12} \\
\frac{d_{1}}{d_{3}}=\operatorname{NC}_{0}^{-1 / 3} & \frac{V_{1}}{V_{3}}=\operatorname{MK}_{0}^{1 / 3}
\end{array}
$$

On peut utiliser l'un quelconque de ces couples de grandeurs $d_{i}, V_{i}$, pour constituer les nombres sans dimensions permettant d'étudier les variations de la vitesse limite d'une bulle en fonction de son diamètre, et c'est ainsi que Wallis [5] a choisi le couple $d_{1}, V_{1}$. Nous avons montré qu'en retenant le couple $d_{2}, V_{2}$, on peut proposer une représentation universelle simple de ces variations.

\section{Bibliographie}

[1] BENFRATELLO G. - Moto di una bolla d'aria entro un liquido in quiete. L'energia electrica ; Part. I. février 1953 , p. $80-97$; Part. II. août 1953, p. 486-512 : Part. III. juillet 1954, p. $481-494$.

[2] HABERMAN (W.B.) et MORTON (R.K.)- An experimental study of bubbles moving in liquids. Am. Soc. of Civil Eng. Transactions (1956) vol. 121, p. 227-252.
[31 WEGENER (P.) et PARLANGE (J.Y.) - Spherical-cap bubbles. Annual Review of Fluid Mechanics, vol. 5 (1973) p. 79-100. Voir aussi Zeits. f. Flugwissenschaft (1971) p. 347-352.

[4] HARPER (J.F.) - The motion of bubbles and drops through liquids. Advances in Applied Mechanics, vol. 12 (1972), p. 59-129

[5] WALLIS (G.B. ) - The terminal speed of single drops of bubbles in an infinite medium. Int. J. Multiphase Flow, vol. I (1974), p. 491-511.

[6] ABRAHAM (F.S. ) - Functional dependance of drag coefficient of a sphere on Reynolds number. The Physics of Fluids (1970), tome 2, p. 2194-2195.

[7] HADAMARD (J.) - Mouvement permanent lent d'une sphère liquide et visqueuse dans un liquide visqueux. C.R. Ac. Sc. t. 152 , (1911), p. 1735-1738; t. 154 (1912), p. 109.

[8] RYBCZYNSKI (W. ) - Sur le mouvement de translation d'une goutte isolée dans un milieu visqueux (en allemand). Bull. Int. Acad. Pol. Sci., Cracovie (1911), Série A, p.4046.

[9] WINNIKOV (S.) et CHAO (B.T.) - Droppiet motion in purified systems. The physics of fluids, vol. 9 (1966), p. 50-61.

[10] GORODETZKAIA. - Vitesse d'ascension des bulles de gaz dans l'eau et dans des solutions aqueuses aux grandes valeurs du nombre de Reynolds (en russe). Revue de Phy. sique et Chimie (1949), tome 23, p. 1-71.

[11] MALENKOV (L.G.) - Motion of large gas bubbles ascending in a liquid. Traduct. du russe dans Journal of Applied Mechanics on Technical Papers (1968), p. 750754.

[12] MENDELSON (H. ) - The prediction of bubble terminal velocities from wave theory, A.I. Ch. E. Journal (1967), vol. 13, p. 250-253.

[13] COMOLET (R. ) - Vitesse d'ascension limite d'une bulle de gaz isolée dans un liquide peu visqueux. Journal de Mécanique Appliquée (1979), à paraître.

[14] COLLAY R. - Etude de la formation et de la vitesse d'ascension d'une bulle d'air dans une colonne d'eau pure. Thèse de spécialité. Paris (1973).

[15] HAGGARD (J.B.) et MASICA (W.J.) - Motion of single bubble under low gravitational conditions. NASA T.N. 5452 (1969).

[16] ROSENBERG (R.) - The drag and shape of air bubbles moving in liquids. Report $\mathrm{N}^{\circ}$ 727. David Taylor Model Basin (1950). 